\title{
Children's physical activity and sedentary time compared using assessments of accelerometry counts and muscle activity level
}

\author{
Ying Gao Corresp.., ${ }^{1}$, Martti Melin ${ }^{1}$ ， Karoliina Mäkäräinen ${ }^{1}$, Timo Rantalainen ${ }^{1}$, Arto Pesola ${ }^{2}$, Arto Laukkanen \\ ${ }^{3}$, Arja Sääkslahti ${ }^{3}$, Taija Finni ${ }^{1}$ \\ ${ }^{1}$ Neuromuscular Research Center, Faculty of Sport and Health Sciences, University of Jyväskylä, Jyväskylä, Finland \\ 2 Active Life Lab, South-Eastern Finland University of Applied Sciences, Mikkeli, Finland \\ 3 Faculty of Sport and Health Sciences, University of Jyväskylä, Jyväskylä, Finland \\ Corresponding Author: Ying Gao \\ Email address: ying.y.gao@jyu.fi
}

Background. This research compared accelerometry (ACC) -derived and muscle electromyography (EMG) -based estimates of physical activity (PA) and sedentary time in typical PA tasks and during daily life of children.

Methods. Data was included from two exploratory studies. In Study I, 6-7-year-old children ( $n=11,64 \%$ girl) were assessed for 8 PA tasks (walking, stair negotiation, climbing, crawling, swinging, balancing, trampoline jumping and a game of tag). In Study II, 7-9-year-old children ( $\mathrm{n}=14,38 \%$ girl) were assessed for 6 PA tasks (walking, sitting, static squat, single leg hops, jump for height and standing long jump), and daily PA during 1 day with and 1 day without structured exercise. Quadriceps and hamstring muscle activity and inactivity using EMG shorts and acceleration by waist-mounted accelerometer were simultaneously measured and classified as sedentary, light, moderate and vigorous activity. Data from ACC was further analyzed using 5 different published cut-off points and varying time windows ( $1-60 \mathrm{~s}$ ) for comparison with EMG.

Results. In the PA tasks ACC counts and EMG amplitude showed marked differences in swinging, trampoline jumping, crawling, static squat, single leg hops, standing long jump and jump for height, the difference being over $170 \%$ when signals were normalized to that during walking. Furthermore, in walking, swinging, trampoline jumping, stair negotiation and crawling ACC classified over $60 \%$ of the time as vigorous-intensity activity, while EMG indicated primarily light- and moderate-intensity activities. During both days with and without exercise, ACC resulted in greater proportion of light activity $(p<0.01)$ and smaller proportion of moderate activity compared to EMG $(p<0.05)$. The choice of cut-off points and epoch length in ACC analysis influenced the classification of PA level and sedentary time. In the analysis of daily activities the cut-off points by Evenson et al. (2008) with epochs of $7.5 \mathrm{~s}$ and $15 \mathrm{~s}$ yielded the smallest difference (less than $10 \%$ of recording time at each intensity) against EMG-derived PA levels.

Discussion. This research provides novel insight on muscle activity and thereby on neuromuscular loading of major locomotor muscles during normal daily activities of children. While EMG and ACC provided similar estimates of sedentary time in 13 typical PA tasks, duration of light, moderate and vigorous PA varied considerably between the methods especially during walking, stair negotiation, crawling, swinging and trampoline jumping. Evenson's (2008) cut-off points with $\leq 15 \mathrm{~s}$ epoch provided similar classification of PA than EMG during daily life. Compared to impacts recorded using ACC, EMG can provide understanding on children's neuromuscular loading during motor tasks that is useful when studying effects of PA interventions on, and development of, motor competence and coordination. 
$1 \quad$ Children's physical activity and sedentary time compared using

3 Ying Gao ${ }^{1}$, Martti Melin ${ }^{1}$, Karoliina Mäkäräinen ${ }^{1}$, Timo Rantalainen ${ }^{1}$, Arto Pesola ${ }^{2}$, Arto

4 Laukkanen $^{3}$, Arja Sääkslahti ${ }^{3}$, Taija Finni ${ }^{1}$

$5{ }^{1}$ Neuromuscular Research Center, Faculty of Sport and Health Sciences, University of

6 Jyväskylä, Jyväskylä, Finland

$7 \quad{ }^{2}$ Active Life Lab, South-Eastern Finland University of Applied Sciences, Mikkeli, Finland

$8{ }^{3}$ Faculty of Sport and Health Sciences, University of Jyväskylä, Jyväskylä, Finland

9

10 Corresponding author:

11 Ying Gao

12 E-mail: ying.y.gao@jyu.fi

13

14

15

16

17

18

19

20

21

22 
24 Acknowledgements: We thank Elina Heikkinen for the help in data collection in Study I.

25 Running title: Accelerometry and EMG in children 


\section{Abstract}

Background. This research compared accelerometry (ACC) -derived and muscle electromyography (EMG) -based estimates of physical activity (PA) and sedentary time in typical PA tasks and during daily life of children.

Methods. Data was included from two exploratory studies. In Study I, 6-7-year-old children $(\mathrm{n}=$ 11, 64\% girl) were assessed for 8 PA tasks (walking, stair negotiation, climbing, crawling, swinging, balancing, trampoline jumping and a game of tag). In Study II, 7-9-year-old children ( $\mathrm{n}=14,38 \%$ girl) were assessed for 6 PA tasks (walking, sitting, static squat, single leg hops, jump for height and standing long jump), and daily PA during 1 day with and 1 day without structured exercise. Quadriceps and hamstring muscle activity and inactivity using EMG shorts and acceleration by waist-mounted accelerometer were simultaneously measured and classified as sedentary, light, moderate and vigorous activity. Data from ACC was further analyzed using 5 different published cut-off points and varying time windows (1-60 s) for comparison with EMG.

Results. In the PA tasks ACC counts and EMG amplitude showed marked differences in swinging, trampoline jumping, crawling, static squat, single leg hops, standing long jump and jump for height, the difference being over $170 \%$ when signals were normalized to that during walking. Furthermore, in walking, swinging, trampoline jumping, stair negotiation and crawling ACC classified over $60 \%$ of the time as vigorous-intensity activity, while EMG indicated primarily light- and moderate-intensity activities. During both days with and without exercise, ACC resulted in greater proportion of light activity $(p<0.01)$ and smaller proportion of moderate activity compared to EMG $(\mathrm{p}<0.05)$. The choice of cut-off points and epoch length in ACC analysis influenced the classification of PA level and sedentary time. In the analysis of daily activities the cut-off points by Evenson et al. (2008) with epochs of $7.5 \mathrm{~s}$ and $15 \mathrm{~s}$ yielded 
49 the smallest difference (less than $10 \%$ of recording time at each intensity) against EMG-derived 50 PA levels.

51 Discussion. This research provides novel insight on muscle activity and thereby on

52 neuromuscular loading of major locomotor muscles during normal daily activities of children.

53 While EMG and ACC provided similar estimates of sedentary time in 13 typical PA tasks,

54 duration of light, moderate and vigorous PA varied considerably between the methods especially

55 during walking, stair negotiation, crawling, swinging and trampoline jumping. Evenson's (2008)

56 cut-off points with $\leq 15 \mathrm{~s}$ epoch provided similar classification of PA than EMG during daily life.

57 Compared to impacts recorded using ACC, EMG can provide understanding on children's

58 neuromuscular loading during motor tasks that is useful when studying effects of PA

59 interventions on, and development of, motor competence and coordination.

60

61

62 
63

64

65

66

67

\section{Introduction}

A physically active childhood enhances physically active lifestyle over the life-span (Telama et al., 2014). It is well documented that physical activity (PA) is associated with numerous physical, psychological/social, and cognitive health benefits and it can reduce several risk factors for chronic diseases, e.g., overweight and obesity (Strong et al., 2005; Poitras et al., 2016). Based on World Health Organization recommendations (2010), children are encouraged to engage in at least 60 minutes per day of moderate-to-vigorous-intensity physical activity (MVPA) (WHO, 2010). However, children spend on average 8 to 9 hours/day in sedentary activities (LeBlanc, Katzmarzyk \& Barreira, 2015) and sedentary behavior (SB) tends to increase $\sim 30$ minutes per day per year in school-aged children (Tanaka, Reilly \& Huang, 2014). A rapidly growing body of evidence shows excessive SB to be linked with the accumulation of various health risks, decreased fitness, low self-esteem and decreased academic achievement in school-aged children and youth (5-17 year-old) (LeBlanc, Katzmarzyk \& Barreira, 2015; Carson et al., 2016). It is therefore necessary to accurately classify both SB and PA profiles of children in order to understand their association with health and wellbeing and to inform future intervention programs and PA guidelines.

Accelerometry (ACC) is widely used for the objective monitoring of PA and sedentary time (Migueles et al., 2017). Typically, ACC is used to detect accelerations via body movements that are converted to a quantifiable measure such as counts (Chen \& Bassett, 2005; Godfrey et al., 2008) or mean amplitude deviation (Vähä-Ypyä et al., 2015), which are validated to estimate metabolic loading of PA. Activities are typically categorized into sedentary, light, moderate and vigorous based on activity counts over epochs of a few seconds to a minute (Migueles et al., 2017). The cut-off points for the various categories are based on metabolic equivalents (MET, 
86 equals to $3.5 \mathrm{ml} / \mathrm{kg} / \mathrm{min}$ ) derived from oxygen uptake measurements ( $\geq 3 \mathrm{METs}$ for moderate, $\geq$ 876 METs for vigorous) or observation of tasks or behaviors with specific METs value (e.g. sitting 88 refers to sedentary, walking to moderate and running to vigorous) (Migueles et al., 2017). In 89 children, however, the use of standard METs as the reference for cut-off points to classify PA 90 intensity has been questioned (Harrell et al., 2005; Ridley \& Olds, 2008; Saint-Maurice et al., 91 2016). Although several different cut-off points have been reported and used in children's studies 92 (Migueles et al., 2017), the established thresholds may vary depending on sample size, age, 93 device version, data processing, reference method and selected tasks, such as in the published 94 methods of Evenson et al. (2008), Van Cauwenberghe et al. (2011), Pate et al. (2006), Puyau et al. (2002) and Sirard et al. (2005). Besides the cut-off values, the epoch length (analysis window 96 duration) and band-pass filters (high and low) for sedentary, light, moderate and vigorous 97 activity may also influence the outcomes and their interpretations in children (Ojiambo et al., 2011). Thus, methodological considerations are meaningful when assessing PA and sedentary time using ACC.

It is important to note that during childhood not only the cardiorespiratory (metabolic) but neuromuscular system in particular plays an important role as a major contributor to the 102 development of motor performance, fitness, as well as proficiency of gross motor skills (Haywood \& Getchell, 2014). Because of the nature of accelerometers and the chosen analysis methods, measuring acceleration does not necessarily reflect neuromuscular loading of PA. Relying on the counts based proxy for metabolic cost likely misclassifies quiet standing as SB (Kozey-Keadle et al., 2011) although standing increases muscle activity and energy expenditure 107 as compared to sitting (Mansoubi et al., 2015; Gao et al., 2017). It is important to note that increases in energy expenditure, when people are physically active, are due to the activation of 
109 skeletal muscles (Caspersen, Powell \& Christenson, 1985). When activated, muscle’s metabolic

110 rate is increased rapidly (and dramatically, 30-50 times of that during resting (McClave \&

111 Snider, 2001; Egan \& Zierath, 2013)) and overall energy expenditure is increased (Gao et al.,

112 2017). On the other hand, lack of muscle contractions (and consequent lack of metabolic

113 stimulus) may be a driver of the aforementioned adverse health outcomes associated with SB,

114 although the possible underlying mechanisms are likely complex and currently unclear

115 (Hamilton, Hamilton \& Zderic, 2007; Hamilton, 2017). Nevertheless, recordings of muscle

116 activity can provide information on the primary stimulus for increased energy expenditure.

117 Electromyography (EMG) can provide complementary and additional information of the entire

118 PA spectrum by quantifying muscle activity during daily activities (Tikkanen et al., 2013, 2014).

119 Specially, we have used novel textile EMG shorts for multiple-day recordings of main locomotor

120 muscle activity and inactivity during normal daily life (Finni et al., 2007, 2014; Tikkanen et al.,

121 2013; Pesola et al., 2015). We have previously reported in adults that EMG shorts predict energy

122 expenditure across a range of PA intensities particularly well when individualized calibrations

123 are used (Tikkanen et al., 2014). Moreover, EMG provides insight into neuromuscular control

124 (Vigotsky et al., 2018), which is essential when studying the development of motor skill

125 competence in childhood (Keawutan et al., 2014). The advantage of EMG lies not only in its

126 nature to reflect the primary response, e.g. muscle's metabolic loading to PA (Kemppainen et al.,

127 2002), but also in the sensitivity of EMG to detect low intensities (Pesola et al., 2016) and

128 typically the instantaneous and sporadic activities of children (Baquet et al., 2007; Laukkanen et

129 al., 2013, 2014; Poitras et al., 2016). Thus, EMG as a measure of muscle activity reflects both

130 metabolic activity of muscles but also characterizes the neuromuscular function. It is expected 
131 that the detailed measurement of muscle activity by using EMG may deepen our understanding

132 of patterns of PA and SB in children.

133 The purpose of this exploratory research was to compare ACC and muscle EMG activity-derived

134 estimates of PA and sedentary time in 13 typical PA tasks and during daily life of children. Due

135 to the nature of ACC to record impacts and EMG to reflect muscle activity, we hypothesized that

136 the mechanical and neuromuscular loading characterized by these different assessment methods

137 categorize several tasks differently in typical PA behaviors of children, and therefore also during

138 daily life. Furthermore, to inform about the selection of cut-off values and epoch lengths for

139 classification of PA intensity levels when using ACC, we compared the outcome of commonly

140 used values to the results obtained using EMG.

\section{Materials \& Methods}

143 This exploratory research included two independent studies, which both assessed different PA 144 tasks occurring typically during children's daily life (Study I \& II). Combining the studies 145 allowed characterization of physiological requirements with the two different methods, ACC and 146 EMG, from total of 13 different tasks typically occurring in children's daily life. Study II further 147 measured children's PA on a day with and on a day without structured exercise allowing 148 methodological comparison when estimating daily PA levels.

\section{Recruitment and study sample}

Study I. A total of 18 first grade children from one central Finland elementary school volunteered

to participate in Study I. Of these participants, $11(6.7 \pm 0.5$ years, 63.6\% girl, height $127.0 \pm 3.6$

$152 \mathrm{~cm}$, body mass $26.6 \pm 2.5 \mathrm{~kg}$, BMI $16.5 \pm 1.6 \mathrm{~kg} / \mathrm{m}^{2}$ ) were included in the final sample. The 
153 reason for exclusion $(n=7)$ was insufficient data containing excessive artefacts in one or more

154 EMG channels.

155 Study II. A total of 14 volunteers $(8.6 \pm 0.8$ years, $35.7 \%$ girl, height $130.9 \pm 8.0 \mathrm{~cm}$, body mass

$15628.1 \pm 4.3 \mathrm{~kg}$, BMI $16.3 \pm 1.3 \mathrm{~kg} / \mathrm{m}^{2}$ ) were recruited from sports clubs in central Finland and

157 were measured over two days. Of these participants, ACC data from 14 and EMG from 12 was

158 included when analyzing the typical PA tasks. During daily life successful recordings using ACC

159 were made in all 14 participants on one day and in 13 participants on both days. EMG recordings

160 were successful for one day in 10 participants and for both days in 7 participants.

161 Both Study I (26.8.2014) and Study II (25.8.2012) received ethics approval from the Ethics

162 Committee of the University of Jyväskylä. During recruitment the study purpose, all the 163 procedures, benefits and risks were explained and children were informed that they could decline

164 from any part of the study without any consequences. All children provided an oral consent and 165 their legal guardians provided written informed consent before any measurements. The studies 166 were conducted in agreement with the Declaration of Helsinki.

\section{Study design and protocol}

EMG and ACC were measured simultaneously during 13 typical PA tasks on weekdays. Study I assessed walking, stair negotiation, climbing, crawling, swinging, balancing, trampoline jumping and game of tag. These assessments were done during afternoon hours. In Study II, assessment

171 of walking, sitting (on the floor), static squat, single leg hops, jump for height and standing long 172 jump were done during morning after breakfast. In addition, Study II further monitored the PA 173 level during one day with and one day without structured exercise that took place in a sports 
174 club. Data during walking, sitting and static squat from five participants were recorded on both

175 days and analyzed for reliability (Supplement: Sheet 6. EMG reliability).

176 In both studies, the participants were first introduced to ACC and EMG devices. Then they were

177 instructed and helped to put on EMG shorts (Myontec Ltd, Kuopio, Finland) and waist-mounted

178 accelerometer (X6-1a, Gulf Coast Data Concepts Inc., Waveland, MS, USA) that was attached

179 using an elastic belt. Both devices were set to record throughout the protocol with synchronizing

180 arranged using the same computer clock. Then the participants were asked to perform the PA

181 tasks according to a standard protocol. The instruction given to the participants is included in

182 Supplement file (Sheet 5. PA task instructions).

183 The average duration of each PA task ranged from 1 second to 2 minutes depending on the type 184 of task (Table 1). The total measurement time was about 30 minutes and participants were given 185 ad libitum rest time between tasks to prevent accumulation of fatigue. All PA tasks were timed 186 and recorded in a $\log$ sheet.

Day with and without structured exercise

188 In Study II, after completing the PA tasks, the devices were left intact to record activities during 189 the remaining day. These day-long recordings were repeated on schooldays that were selected to 190 be as similar as possible (e.g. neither of days or both days included physical education, same 191 duration of school day) with the exception that one day included structured exercise in a sports 192 club. The exercise training (football or floorball) lasted 60-90 minutes during the 193 afternoon/evening hours. The participants were asked to remove the recording devices in the 194 evening.

\section{Recordings and analysis}


196 Full duration of the PA tasks (Table 1) was analyzed for both ACC and EMG. In ACC analysis a $1971 \mathrm{~s}$ epoch was used. In tasks of single leg hops, jump for height and standing long jump, both 198 push-off and landing were included in the analysis.

199 For the day-long recordings analysis was performed on about 9 hour period at the same time of 200 the day during both days with and without exercise. In case recordings on one day were shorter 201 than on the other, this procedure eliminated problems in comparing the days but shortened the 202 evaluated time slightly. Successful simultaneous ACC and EMG recordings were required for 203 inclusion into analysis.

204 Accelerometry. A triaxial accelerometer ( \pm 6 g, 16-bit A/D conversion, sampling at $40 \mathrm{~Hz}$ ) was 205 secured onto the anterior waistline with an elastic belt at the level of the L4-L5. The resultant 206 vector $\sqrt{x^{2}+y^{2}+z^{2}}$ of the triaxial accelerometer signal was calculated, band pass filtered $(0.25$ $207 \mathrm{~Hz}$ to $11 \mathrm{~Hz}$ ), and a $0.05 \mathrm{~g}$ dead-band was digitally applied. Integration and filtering of 208 accelerometer signals were performed in MATLAB software (MathWorks, MA, USA) and 209 converted to values corresponding to ActiGraph GT3X (Laukkanen et al., 2014). The conversion 210 factor was obtained from simultaneous recordings with both X6-1a and ActiGraph GT3X 211 devices in children (Laukkanen et al., 2014). Data were summed over 15 second epochs with the 212 mean of accelerometer counts less than 12 counts classified as sedentary time, $12-508$ counts as 213 light-intensity activity, 508-719 counts as moderate-intensity activity, and more than 719 counts 214 as vigorous-intensity activity (Evenson et al., 2008, thresholds for multi-axial accelerometer). To 215 account for epoch length effects, data were also summed over either in $1 \mathrm{~s}, 7.5 \mathrm{~s}, 30 \mathrm{~s}$ or $60 \mathrm{~s}$ 216 using time-window accommodated thresholds from Evenson et al. (2008) (e.g. for sedentary 217 threshold $1 \mathrm{~s}$ is 0.8 counts; $7.5 \mathrm{~s}$ is 6 counts, $15 \mathrm{~s}$ is 12 counts, $30 \mathrm{~s}$ is 24 counts, $60 \mathrm{~s}$ is 48 218 counts). Furthermore, the data was analyzed using $15 \mathrm{~s}$ epochs and cut-off thresholds from Van 
219 Cauwenberghe et al. (2010), Pate et al. (2006), Puyau et al. (2002) and Sirard et al. (2005) (Table

220 2). These methods were chosen because they are most often used in children's PA research

221 (Migueles et al., 2017), but they rely on different criterion measures (e.g. oxygen uptake or

222 observation) and sample (e.g. different age and population). To allow comparison with EMG,

223 ACC counts were normalized to walking (e.g. counts value during walking was considered as $224100 \%)$.

225 Textile EMG shorts were used to measure muscle activities from the quadriceps and the 226 hamstring muscles. The shorts were made of knitted fabric similar to elastic clothes, into which 227 textile EMG electrodes were embedded in order to measure EMG from the skin surface. 228 Electrodes were positioned in a bipolar configuration over the muscle bellies of the left and right quadriceps (the conductive area of $18 \mathrm{~cm}^{2}$ ) and hamstring muscles (the conductive area of 12 $230 \mathrm{~cm}^{2}$ ). Reference electrodes (the conductive area of $22 \mathrm{~cm}^{2}$ ) were located longitudinally over tractus iliotibialis. Electrode paste (Redux Electrolyte Crème, Parker, Inc., Fairfield, NJ) was used to minimize the skin-electrode impedance. The device applied an analog bandpass filter (50 $\mathrm{Hz}$ to $200 \mathrm{~Hz}$ ), and sampled at $1000 \mathrm{~Hz}$ after which pre-processed the data into non-overlapping $40 \mathrm{~ms}$ root-mean-squared values, which are stored in a small waist-mounted module (Finni et al., 235 2007). These EMG shorts have been tested in adults for validity, repeatability and feasibility, and a detailed description of the recording devices has been reported (Finni et al., 2007; Pesola et al., 2014). In the present study the day-to-day reliability was assessed in 5 participants and found to be good (Supplement: Sheet 6. EMG reliability). The EMG signals were normalized channel by channel to EMG values measured during normal walking in both Study I and Study II. The normalized EMG from right and left legs were averaged from analysis window to produce the mean quadriceps and hamstring muscle EMG, and further averaged to produce the mean thigh 
242 muscle EMG. Classification into different PA categories was based on individually obtained

243 EMG values: 1) EMG amplitude $<3 \mu \mathrm{V}$ was classified as muscle inactivity, 2) amplitudes

244 between $3 \mu \mathrm{V}$ and the mean EMG value during normal walking as light activity, 3) amplitudes

245 between mean EMG during walking and 2*mean EMG during walking as moderate activity, and

246 4) amplitudes above $2 *$ mean EMG during walking as vigorous activity (Tikkanen et al., 2013;

247 Pesola et al., 2015, 2016).

248 Statistical analysis

249 Statistical analyses were conducted using IBM SPSS for Windows 22.0 (SPSS Inc., Chicago, IL,

250 USA). Values are reported as means \pm standard deviations unless otherwise indicated. Tests of

251 normality (Shapiro-Wilk) were applied. Repeated measures analysis of variance (ANOVA) was

252 used to compare the main effects of day (exercise vs. no exercise) and device (ACC vs. EMG)

253 and their interaction. ANOVA was also used to examine days with and without exercise

254 separately to examine whether there are main effects of intensity (sedentary, light, moderate,

255 vigorous) and device (ACC vs. EMG), and their interaction. Comparison of EMG and different

256 ACC analysis methods (Evenson et al. 2008, Puyau et al., 2002; Sirard et al., 2005; Pate et al.,

257 2006; Van Cauwenberghe et al., 2011) were also examined with ANOVA and main effects of the

258 method and intensity and their interaction were examined. Similarly, ANOVA was applied to

259 test significant differences and interaction between epoch length (Evenson's method with 1 s, 7.5

260 s, 15 s, 30 s, 60 s and EMG) and intensity. Violations of sphericity were corrected using Huynh-

261 Feldt correction and ANOVA was followed by Bonferroni's post hoc for multiple comparisons if

262 necessary to localize the difference. During children's daily life, agreement between ACC and

263 EMG was evaluated using the Bland-Altman method (Bland \& Altman, 1986) and for each PA

264 intensity Pearson's correlation coefficients were calculated in order to check if there was 
265 heteroscedasticity. A probability level of $\mathrm{p}<0.05$ (two-tailed) was considered statistically

266 significant.

267

268 Results

269 ACC counts vs. EMG amplitude in PA tasks

270 In order to compare ACC counts and EMG amplitude, both were presented relative to normal

271 walking, where ACC corresponded to an average of 1218 counts (SD 362, range 678-2033).

272 Figure 1 shows ACC counts and EMG amplitude in various PA tasks when normalized to

273 walking. In swinging, trampoline jumping, crawling, static squat, standing long jump, single leg

274 hops and jump for height, there were marked differences (over 170\%) between normalized ACC

275 and EMG values with a range of $-590 \%-1010 \%$. The mean ACC counts ranged from 18 counts

276 in sitting to 13566 counts in trampoline jumping. The range of the normalized EMG amplitude

277 was from $16 \%$ in sitting to $607 \%$ in jumping for height.

278 PA levels in typical PA tasks

279 Figure 2 shows the distribution of PA intensities for each task when assessed using ACC and

280 EMG. EMG-derived muscle inactivity time (range of $0.0 \%-0.5 \%$ ) was similar to sedentary time

281 assessed using ACC (range of $0.2 \%-6.3 \%$ ). Duration of light-, moderate- and vigorous intensity

282 varied considerably between ACC and EMG in several PA tasks. Walking, stair negotiation,

283 crawling, swinging, trampoline jumping and game of tag were all activities where ACC showed

284 that over $60 \%$ of the time was vigorous activity. However, EMG showed that none of the

285 activities reached such high proportion (over $60 \%$ of time) of vigorous activity. 
287 The comparison of PA levels on days with and without exercise is shown in Table 3. ANOVA

288 showed no main effects of day or device, or interaction. When the days were examined

289 separately, both a day with exercise and a day without exercise revealed significant main effects

290 for device $(\mathrm{p}=0.001$ and $\mathrm{p}=0.032$, respectively) and intensity ( $\mathrm{p}<0.001$ for both days) and

291 device*intensity interaction $(\mathrm{p}=0.007$ and $\mathrm{p}=0.013$, respectively). Post hoc analysis revealed

292 that time spent in light activity $(\mathrm{p}=0.001$ and $\mathrm{p}=0.002)$ and moderate activity $(\mathrm{p}=0.031$ and $\mathrm{p}$

$293=0.029)$ were significantly different between the methods on days with and without exercise,

294 respectively (Table 3). On average, ACC yielded 9.4\% (p<0.001) greater amount of light 295 activity but 5.8\% ( $<0.001)$ lower amount of moderate activity (Figure 3$)$. Average time spent 296 in sedentary or vigorous activity did not differ between ACC and EMG methods (Figure 3).

297 Bland-Altman plots further revealed heteroscedasticity in all but light PA categories so that the 298 difference between the methods became accentuated with greater amount of time spent at the 299 given category (Figure 3).

\section{Comparison of different thresholds in ACC and EMG}

301 Since the day with or without exercise had similar PA levels, average of the days was used to 302 compare different cut-off points in ACC and EMG estimated sedentary (/muscle inactivity), light, moderate and vigorous activity time. Figure 4 shows comparison of EMG and differently and intensity $(\mathrm{p}<0.001)$ with interaction $(\mathrm{p}<0.001)$. When compared to EMG, Evenson's

306 (Evenson et al., 2008) and Pate's (Pate et al., 2006) cut-off points yielded the smallest 
310 and vigorous activity time (n.s.) as EMG. All other methods showed smaller light activity time (p

$311<0.001)$ compared to EMG. Moderate activity time was similar to EMG with Pate's methods,

312 while with others being significantly different from EMG ( $\mathrm{p} \leq 0.008)$. Vigorous activity using

313 Puyau's $(\mathrm{p}=0.010)$ and Sirard's methods $(\mathrm{p}=0.016)$ was significantly smaller than using EMG,

314 other methods did not show significant differences.

315

316 In the epoch-length analysis using Evenson's cut-off points and EMG we found main effect of

317 epoch $(\mathrm{p}<0.001)$ and intensity $(\mathrm{p}<0.001)$ with interaction $(\mathrm{p}<0.001)$. There was a progressive

318 decrease in the time spent in sedentary $(56 \%$ to $36 \%, p<0.001)$ and increase in light-intensity

319 activity $(34 \%$ to $54 \%, \mathrm{p}<0.001)$ as the epoch length increased from $1 \mathrm{~s}$ to $30 \mathrm{~s}$ (Figure 5). The

320 time spent in moderate to vigorous intensity activity was remained at about $10 \%$ of recording

321 time between the 1-30 s epochs. With $60 \mathrm{~s}$ epoch the time spent in sedentary and light intensity

322 activity dominated. Overall, the epochs of $7.5 \mathrm{~s}$ and $15 \mathrm{~s}$ showed the least difference with EMG

323 method (less than $10 \%$ at each intensity level where the only significant differences were at

324 moderate intensity, $\mathrm{p}=0.004$ and $\mathrm{p}=0.008$, respectively).

\section{Discussion}

326 The primary findings of this exploratory study were as follows. Firstly, in accordance with the

327 hypothesis, ACC and EMG provided different results and interpretation of the intensity and

328 duration of children's typical PA tasks. Muscle EMG activity was emphasised over ACC counts

329 during crawling, static squat, single leg hops, standing long jump and jump for height, while

330 ACC values were emphasised over EMG during swinging and trampoline jumping. During

331 walking, swinging, trampoline jumping, stair negotiation and crawling ACC showed that over $60 \%$

332 of the time was spent in vigorous intensity while EMG allocated a high proportion of the time to 
333 light and moderate intensity. Secondly, during normal daily life ACC resulted in greater

334 proportion of light activity but smaller proportion of moderate activity compared to EMG.

335 Amongst the different thresholds (Puyau et al., 2002; Sirard et al., 2005; Pate et al., 2006; Van

336 Cauwenberghe et al., 2011), the cut-off points by Evenson et al. (2008) with epochs of $7.5 \mathrm{~s}$ and

33715 s yielded the smallest difference against EMG-derived PA levels.

338 Although ACC and EMG inherently measure different quantities, their assessments are

339 interrelated. In the present study, we assessed a total of 13 different typical daily tasks with

340 simultaneous measurements of ACC counts and EMG amplitude. When ACC and EMG values

341 were both normalized to walking, the difference between ACC and EMG had the smallest

342 difference during sitting, climbing, stair negotiation and game of tag (range of $14 \%-47 \%$ ) as

343 compared to the other tasks (Figure 1). Specially, in crawling, static squat, standing long jump,

344 single leg hops and jump for height, EMG indicated over 170\% higher relative intensity

345 compared to ACC, while in swinging and trampoline jumping, the roles were reversed. This is

346 reasonable since in trampoline jumping and swinging the body is hurled through the space

347 resulting in high ACC counts, while only brief bursts of main locomotor muscle activity is

348 observed in the EMG. On the other hand, in balancing and static squat, the body is quasi

349 stationary while the main locomotor muscles are working to maintain the posture position.

350 Predictably, this results in low ACC counts, while EMG activity is observed. The distribution of

351 intensity levels in the typical PA tasks further highlights the methodological differences (Figure 352 2).

353 In case of sporadic activities, EMG presumably results in a more realistic representation of 354 energy demands while ACC counts summed over the duration dilute the effect. For example, 355 during jumping tasks high EMG amplitudes in both push-off and landing phases are necessary, 
356 and thus resulted in a great mean EMG. Contrarily, ACC counts were relatively low during

357 standing long jump, single leg hops, and jump for height because high ACC peaks were only

358 seen in during landing. Thus, it suggested that further insight could be achieved by comparing

359 the counts with g-value analysis where raw ACC data provides more direct information of the

360 impacts loading the body (Laukkanen et al., 2013, 2014).

361 Increasing participation in organized PA has been suggested as a strategy for increasing overall

362 PA in children (Hebert et al., 2015). However, in the present study with small sample, we did not

363 find significant difference in either EMG or ACC PA levels between days with and without

364 exercise (Table 3). It seems that one session of 60-90 minutes organized exercise may not alter

365 the PA level of the whole day, although it provides a supportive environment for increasing PA

366 during the organized session. Children may compensate their increased PA by relaxing outside

367 the exercise session, and transportation to the exercise venue (especially during winter when

368 some assessments were done) may require sedentary time that is not present on days without

369 exercise (Ridley, Zabeen \& Lunnay, 2018).

370 During the $9 \mathrm{~h}$ recordings, ACC and EMG provided slightly different values for the time spent at

371 different PA intensities. While there were no significant differences between ACC estimated

372 sedentary time and EMG estimated muscle inactivity time on either day with or without exercise,

373 ACC yielded $\sim 9 \%$ more light PA time but $\sim 5 \%$ less moderate activity time than EMG (Table 3 ).

374 Furthermore, the Bland-Altman plots showed heteroscedasticity for sedentary, moderate and

375 vigorous activity time so that the difference between ACC and EMG became accentuated with

376 greater amount of time spent at the given category (Figure 3). Consequently, the choice of

377 method influences interpretation; in the present sample, EMG allows the conclusion that the

378 children meet PA recommendations having over 60 minutes MVPA while this was not reached 
379 according to ACC. Note, that the recordings were only $9 \mathrm{~h}$ and if the data was adjusted for $12 \mathrm{~h}$,

380 for example, also ACC data suggests that these children meet PA recommendations.

381 In this context, it is important to discuss the chosen EMG thresholds that were defined on

382 individual basis while the ACC thresholds were the same for all individuals. Firstly, regarding

383 EMG, the data requires normalization in order to compare amplitudes between individuals

384 (Vigotsky et al., 2018). In this study, we normalized the EMG amplitude to that found during

385 normal walking. Furthermore, the thresholds for moderate and vigorous PA levels were

386 associated to individually chosen preferred walking speed of each child. While differences in the

387 walking technique or speed may have influenced the EMG thresholds (Lee \& Hidler, 2008),

388 these differences do not pertain to ACC thresholds. In ACC count vs. EMG amplitude

389 comparison both signals were normalized to walking, which enabled direct comparison in

390 various PA tasks (Figure 1). During walking, mean of 1218 ACC counts were recorded during

391 walking, which is closer to moderate-to-vigorous ACC threshold than light-to-moderate ACC

392 threshold and thus corresponds rather closely with the EMG threshold. On the other hand, the

393 threshold for sedentary time was absolute EMG amplitude and during sitting it was $16 \%$ of

394 walking. The corresponding mean ACC counts value was 18 during sitting that would have been

395 categorized to light activity. This may be due to the fact that the children were sitting on the floor

396 instead of chair in the current study. Furthermore, because this is the first study using EMG

397 shorts in children, more validation work is required to inform of accurate, EMG-derived

398 estimates of PA levels and their thresholds in children. In adults, the validation of muscle

399 inactivity threshold is based on differentiating sitting from standing (Pesola et al., 2014, 2016;

400 Gao et al., 2016). In this study we aimed to differentiate muscle activity from inactivity, and set

401 the threshold $(3 \mu \mathrm{V})$ above signal baseline. This enabled recording of the smallest muscle 
402 activities also when sitting (Pesola et al., 2016), which is the strength of EMG. However, the

403 definition and operationalization of muscle inactivity should be further validated in children.

404 It can be questioned whether comparison of methods that reflect different aspects of PA is 405 reasonable in at the first place. However, PA is defined as body movement caused by muscle 406 activity (Caspersen, Powell \& Christenson, 1985), which supports the use of EMG to measure 407 PA. Both metabolic and neuromuscular loading during PA are associated with specific benefits 408 (Strong et al., 2005; Keawutan et al., 2014; Poitras et al., 2016). In many studies the PA exposure 409 is measured with ACC, but the analyzed outcomes are more related to neuromuscular than 410 metabolic loading of activity, e.g. motor skill competence (Stodden et al., 2008; Lopes et al., 411 2012; Robinson et al., 2015). Therefore, it is important to understand how ACC-derived PA 412 relates to neuromuscular loading and the current study provides data to compare ACC data to 413 directly measured muscle EMG activity.

414 Different cut-off points and epoch lengths were also compared in the present study. As expected, 415 the choice of cut-off points and epoch lengths had significant effects on the time spent in 416 sedentary, light, moderate and vigorous activities. For example, there was a progressive increase 417 in the time spent in sedentary when ACC counts for sedentary is increased from 12 counts 418 (Evenson et al., 2008) to 400 counts (Sirard et al., 2005). However, it should be noted that the 419 published cut-off points were established in their own calibration study. It is recommended to 420 follow the same criterion measures used in original study in order to make comparable and 421 accurate results for the specific data sets (Migueles et al., 2017). The present study aimed to 422 compare ACC with EMG-derived estimates of PA level. We found that Evenson's cut-off points 423 (Evenson et al., 2008) yielded the smallest differences among other methods (Puyau et al., 2002; 424 Sirard et al., 2005; Pate et al., 2006; Van Cauwenberghe et al., 2011) against EMG estimates. 
425 Since Evenson's cut-off points have been often used in children's studies (Migueles et al., 2017),

426 it may serve as a surrogate method for EMG-derived estimates of muscle activity and inactivity

427 time in young children.

428 Epoch lengths from $1 \mathrm{~s}$ to $60 \mathrm{~s}$ were explored using the Evenson's cut-off points (Evenson et al., 429 2008). We found a progressive decrease in the time spent in sedentary and increase in light430 intensity as the epoch length was increased from $1 \mathrm{~s}$ to $30 \mathrm{~s}$. The proportion of moderate- and 431 vigorous-intensity activity were similar for the epoch lengths between $1 \mathrm{~s}$ and $30 \mathrm{~s}$, but for the 432 epoch length of $60 \mathrm{~s}$ the recording time involved mainly sedentary and light activity time. These 433 observations may reflect children's typical sporadic activities and intermittent activity patterns 434 during their daily life (Fischer et al., 2012; Orme et al., 2014), that are diluted with longer epochs. 435 Previous study also compared different epoch lengths, and they found that using shorter epoch 436 lengths (e.g. $15 \mathrm{~s}$ ) compared with $60 \mathrm{~s}$ resulted in less sedentary time and higher MVPA time in 437 children (Ojiambo et al., 2011). It seems that shorter epochs are recommended to capture short 438 bouts of activity occurring frequently in young people (Migueles 2017). Bailey et al. (1995) 439 reported the majority of children's PA bouts occur in a short bouts. Furthermore, when compared 440 with EMG-derived muscle activity levels, the epoch lengths of $7.5 \mathrm{~s}$ and $15 \mathrm{~s}$ had smallest 441 difference in the classification of PA levels, in which the range within $10 \%$ difference for each 442 intensity level. Given that epoch length can influence the outcomes, it is important to use the 443 same epoch length in order to compare different studies. Thus, based on the current findings, 444 future study may use the similar ACC epoch length of $7.5 \mathrm{~s}$ or $15 \mathrm{~s}$ for close association with 445 muscle activity -based estimations of PA levels in children. However, it should be noted that the 446 PA tasks analyzed had different durations. This was chosen so that in each task we could include 447 sufficient number of repetitions (e.g. gait cycle, crawling cycle swing cycle, trampoline jump 
448 cycle) in order to provide accurate representation of the task in different PA intensities. Naturally

449 the cycle time is shorter in gait compared to swinging thus influencing the chosen duration. On

450 the other hand, some tasks are more difficult for children to sustain, such as balancing task or

451 maximal jump, where the time window for constant movement pattern is shorter. Consequently,

452 we accepted variable durations of the analyzed tasks (Table 1).

453 Some limitations should be taken into consideration when interpreting the present results. First,

454 the present research consists of two independent studies with a small sample size that may not be 455 representative; age, motor coordination, body mass, lean body mass, fat mass, height or leg 456 length, for example, may influence accelerations detected by the accelerometer and muscle 457 activities assessed by EMG (Sirard et al., 2005; Pesola et al., 2016). Secondly, selection of the 458 specific thresholds of EMG for muscle inactivity and activity levels affect the results because of 459 the sensitivity of thresholds (Klein et al., 2010). When determining EMG thresholds we have 460 relied on our experience with adults (Tikkanen et al., 2013, 2014; Pesola et al., 2016) but in 461 children, the EMG thresholds require a proper validation study with methodological comparisons, 462 which is currently ongoing. Regarding methodological issues related to comparison of ACC and 463 EMG signal processing, it is to be noted that attempts to process the raw signals in a similar 464 manner should be made to allow more comprehensive methodological comparison and outcome 465 characterization. Furthermore, we compared published cut-off points of ACC in our dataset, 466 although our device and analysis process differed from the data collection procedures and 467 processing criteria used in the original validation/calibration study. Overall, further studies 468 should evaluate children's PA using various methodological approaches to gain understanding of 469 not only the PA behavior but also of the significance of neuromuscular loading during 470 development. 


\section{Conclusions}

472 By comparing accelerometer-derived and muscle EMG activity -based estimates of PA intensity

473 and sedentary time this exploratory research provides novel insight into neuromuscular and

474 metabolic loading of various PA tasks and daily life of school-aged children. In particular,

475 requirement of neuromuscular activity during PA tasks involving quasi static movements

476 (balancing, crawling and static squat), sporadic activities (standing long jump, single leg hops,

477 and jump for height) or movements associated with aerial maneuvers (trampoline jumping,

478 swinging), seem to be poorly represented with ACC counts as compared to EMG. Exploration of 479 different cut-off points and epoch lengths showed that Evenson's (Evenson et al., 2008)

480 thresholds with $\leqslant 15$ s epochs may best mimick EMG activity intensity categories, although

481 during daily life ACC showed more time spent at light and less time at moderate intensity level

482 than EMG. Since EMG and ACC inherently measure different aspects of children's PA, their

483 combination can provide understanding on the quality of PA from both the neuromuscular

484 (development of motor competence and coordination) and metabolic loading (overall movement

485 and energy expenditure) perspectives. 
486

487

488

489

490

491

492

493

494

495

496

497

498

499

500

501

502

503

504

505

506

507

508

509

510

\section{References}

Bailey RC., Olson J., Pepper SL., Porszasz J., Barstow TJ., Cooper DM. 1995. The level and tempo of children's physical activities: an observational study. Medicine and Science in Sports and Exercise $27: 1033-41$.

Baquet G., Stratton G., Van Praagh E., Berthoin S. 2007. Improving physical activity assessment in prepubertal children with high-frequency accelerometry monitoring: A methodological issue. Preventive Medicine 44:143-147. DOI: 10.1016/J.YPMED.2006.10.004.

Bland JM., Altman D. 1986. Statistical methods for assessing agreement between two methods of clinical measurement. The Lancet 327:307-310. DOI: 10.1016/S0140-6736(86)90837-8.

Carson V., Hunter S., Kuzik N., Gray CE., Poitras VJ., Chaput J-P., Saunders TJ., Katzmarzyk PT., Okely AD., Connor Gorber S., Kho ME., Sampson M., Lee H., Tremblay MS., Carson V., Hunter S., Kuzik N., Lee H., Gray C., Poitras V., Chaput J., Sampson M., Tremblay M., Saunders T., Katzmarzyk Pennington P., Okely Early A., Connor Gorber S., Kho M. 2016. Systematic review of sedentary behaviour and health indicators in school-aged children and youth: an update 1. Appl. Physiol. Nutr. Metab. Appl. Physiol. Nutr. Metab. Downloaded from 41:240-265. DOI: 10.1139/apnm-2015-0630.

Caspersen CJ., Powell KE., Christenson GM. 1985. Physical activity, exercise, and physical fitness: definitions and distinctions for health-related research. Public Health Reports 100:126-31.

Van Cauwenberghe E., Labarque V., Trost SG., De Bourdeaudhuij I., Cardon G. 2011. Calibration and comparison of accelerometer cut points in preschool children. International Journal of Pediatric Obesity 6:582-589. DOI: 10.3109/17477166.2010.526223.

Chen KY., Bassett DR. 2005. The Technology of Accelerometry-Based Activity Monitors: Current and Future. Medicine \& Science in Sports \& Exercise 37:S490-S500. DOI: 10.1249/01.mss.0000185571.49104.82.

Egan B., Zierath JR. 2013. Exercise metabolism and the molecular regulation of skeletal muscle 
512 Evenson KR., Catellier DJ., Gill K., Ondrak KS., McMurray RG. 2008. Calibration of two objective 513 measures of physical activity for children. Journal of Sports Sciences 26:1557-1565. DOI:

$514 \quad 10.1080 / 02640410802334196$.

515 Finni T., Haakana P., Pesola AJ., Pullinen T. 2014. Exercise for fitness does not decrease the muscular 516 inactivity time during normal daily life. Scandinavian Journal of Medicine \& Science in Sports $517 \quad 24: 211-9$. DOI: $10.1111 /$ j.1600-0838.2012.01456.x.

518 Finni T., Hu M., Kettunen P., Vilavuo T., Cheng S. 2007. Measurement of EMG activity with textile 519 electrodes embedded into clothing. Physiological Measurement 28:1405-1419. DOI: 10.1088/0967$520 \quad 3334 / 28 / 11 / 007$.

521 Fischer C., Yıldırım M., Salmon J., Chinapaw MJM. 2012. Comparing Different Accelerometer Cut522 Points for Sedentary Time in Children. Pediatric Exercise Science 24:220-228. DOI: $523 \quad 10.1123 /$ pes.24.2.220.

524 Gao Y., Cronin NJ., Pesola AJ., Finni T. 2016. Muscle activity patterns and spinal shrinkage in office 525 workers using a sit-stand workstation versus a sit workstation. Ergonomics 59:1267-1274. DOI: 10.1080/00140139.2016.1139750.

527 Gao Y., Silvennoinen M., Pesola AJ., Kainulainen H., Cronin NJ., Finni T. 2017. Acute Metabolic 528 Response, Energy Expenditure, and EMG Activity in Sitting and Standing. Medicine \& Science in 529 Sports \& Exercise 49:1927-1934. DOI: 10.1249/MSS.0000000000001305.

530 Godfrey A., Conway R., Meagher D., ÓLaighin G. 2008. Direct measurement of human movement by $531 \quad$ accelerometry. Medical Engineering and Physics 30:1364-1386. DOI:

$532 \quad$ 10.1016/j.medengphy.2008.09.005.

533 Hamilton MT. 2017. The role of skeletal muscle contractile duration throughout the whole day: reducing 534 sedentary time and promoting universal physical activity in all people. The Journal of Physiology. $535 \quad$ DOI: $10.1113 / J P 273284$.

536 Hamilton M., Hamilton D., Zderic T. 2007. Role of low energy expenditure and sitting in obesity, 
metabolic syndrome, type 2 diabetes, and cardiovascular disease. Diabetes 56:2655-2667. DOI: 10.2337/db07-0882.CVD.

539

Harrell JS., McMurray RG., Baggett CD., Pennell ML., Pearce PF., Bangdiwala SI. 2005. Energy costs of physical activities in children and adolescents. Medicine and Science in Sports and Exercise $37: 329-36$.

Haywood K., Getchell N. 2014. Life span motor development. Champaign, IL: Human Kinetics.

Hebert JJ., Møller NC., Andersen LB., Wedderkopp N. 2015. Organized sport participation is associated with higher levels of overall health-related physical activity in children (CHAMPS study-DK). PLoS ONE 10:1-12. DOI: 10.1371/journal.pone.0134621.

Keawutan P., Bell K., Davies PSW., Boyd RN. 2014. Systematic review of the relationship between habitual physical activity and motor capacity in children with cerebral palsy. Research in Developmental Disabilities 35:1301-1309. DOI: 10.1016/j.ridd.2014.03.028.

Kemppainen J., Fujimoto T., Kalliokoski KK., Viljanen T., Nuutila P., Knuuti J. 2002. Myocardial and skeletal muscle glucose uptake during exercise in humans. The Journal of Physiology 542:403-12.

Klein CS., Peterson LB., Ferrell S., Thomas CK. 2010. Sensitivity of 24-h EMG duration and intensity in the human vastus lateralis muscle to threshold changes. Journal of Applied Physiology (Bethesda, Md. : 1985) 108:655-61. DOI: 10.1152/japplphysiol.00757.2009.

Kozey-Keadle S., Libertine A., Lyden K., Staudenmayer J., Freedson PS. 2011. Validation of wearable monitors for assessing sedentary behavior. Medicine and Science in Sports and Exercise 43:15611567. DOI: 10.1249/MSS.0b013e31820ce174.

Laukkanen A., Finni T., Pesola A., Sääkslahti A. 2013. Reipas Liikunta Takaa Lasten Motoristen Perustaitojen Kehityksen - Mutta Kevyttäkin Tarvitaan ! 35:47-52.

Laukkanen A., Pesola A., Havu M., Sääkslahti A., Finni T. 2014. Relationship between habitual physical activity and gross motor skills is multifaceted in 5- to 8-year-old children. Scandinavian Journal of Medicine \& Science in Sports 24:e102-e110. DOI: 10.1111/sms.12116.

LeBlanc AG., Katzmarzyk PT., Barreira TV. . . et. al. 2015. Correlates of total sedentary time and screen 
time in 9-11 year-old children around the world: The international study of childhood obesity, lifestyle and the environment. PLoS ONE 10:1-20. DOI: 10.1371/journal.pone.0129622.

565

Lee SJ., Hidler J. 2008. Biomechanics of overground vs. treadmill walking in healthy individuals. Journal of Applied Physiology (Bethesda, Md. : 1985) 104:747-55. DOI: 10.1152/japplphysiol.01380.2006.

Lopes VP., Stodden DF., Bianchi MM., Maia JAR., Rodrigues LP. 2012. Correlation between BMI and motor coordination in children. Journal of Science and Medicine in Sport 15:38-43. DOI: 10.1016/j.jsams.2011.07.005.

Mansoubi M., Pearson N., Clemes S a., Biddle SJ., Bodicoat DH., Tolfrey K., Edwardson CL., Yates T. 2015. Energy expenditure during common sitting and standing tasks: examining the 1.5 MET definition of sedentary behaviour. BMC Public Health 15:516. DOI: 10.1186/s12889-015-1851-x.

McClave SA., Snider HL. 2001. Dissecting the energy needs of the body. Current Opinion in Clinical Nutrition and Metabolic Care 4:143-147. DOI: 10.1097/00075197-200103000-00011.

Migueles JH., Cadenas-Sanchez C., Ekelund U., Delisle Nyström C., Mora-Gonzalez J., Löf M., Labayen I., Ruiz JR., Ortega FB. 2017. Accelerometer Data Collection and Processing Criteria to Assess Physical Activity and Other Outcomes: A Systematic Review and Practical Considerations. Sports Medicine 47:1821-1845. DOI: 10.1007/s40279-017-0716-0.

Ojiambo R., Cuthill R., Budd H., Konstabel K., Casajús JA., González-Agüero A., Anjila E., Reilly JJ., Easton C., Pitsiladis YP. 2011. Impact of methodological decisions on accelerometer outcome variables in young children. International Journal of Obesity 35:S98-S103. DOI: 10.1038/ijo.2011.40.

Orme M., Wijndaele K., Sharp SJ., Westgate K., Ekelund U., Brage S. 2014. Combined influence of epoch length, cut-point and bout duration on accelerometry-derived physical activity. International Journal of Behavioral Nutrition and Physical Activity 11:34. DOI: 10.1186/1479-5868-11-34.

Pate RR., Almeida MJ., McIver KL., Pfeiffer KA., Dowda M. 2006. Validation and calibration of an accelerometer in preschool children. Obesity 14:2000-2006. DOI: 10.1038/oby.2006.234.

Pesola AJ., Laukkanen A., Haakana P., Havu M., Sääkslahti A., Sipilä S., Finni T. 2014. Muscle 

inactivity and activity patterns after sedentary time-targeted randomized controlled trial. Medicine and Science in Sports and Exercise 46:2122-2131. DOI: 10.1249/MSS.0000000000000335.

591

592

593

Pesola AJ., Laukkanen A., Tikkanen O., Finni T. 2016. Heterogeneity of Muscle Activity during Sedentary Behavior. Applied Physiology, Nutrition, and Metabolism 41:1155-62. DOI: 10.1139/apnm-2016-0170.

Pesola AJ., Laukkanen A., Tikkanen O., Sipilä S., Kainulainen H., Finni T. 2015. Muscle inactivity is adversely associated with biomarkers in physically active adults. Medicine and Science in Sports and Exercise 47:1188-1196. DOI: 10.1249/MSS.0000000000000527.

Poitras VJ., Gray CE., Borghese MM., Carson V., Chaput J-P., Janssen I., Katzmarzyk PT., Pate RR., Connor Gorber S., Kho ME., Sampson M., Tremblay MS. 2016. Systematic review of the relationships between objectively measured physical activity and health indicators in school-aged children and youth. Applied Physiology, Nutrition, and Metabolism 41:S197-S239. DOI: 10.1139/apnm-2015-0663.

Puyau MR., Adolph AL., Vohra FA., Butte NF. 2002. Validation and Calibration of Physical Activity Monitors in Children. Obesity Research 10:150-157. DOI: 10.1038/oby.2002.24.

Ridley K., Olds TS. 2008. Assigning Energy Costs to Activities in Children. Medicine \& Science in Sports \& Exercise 40:1439-1446. DOI: 10.1249/MSS.0b013e31817279ef.

Ridley K., Zabeen S., Lunnay BK. 2018. Children's physical activity levels during organised sports practices. Journal of Science and Medicine in Sport. DOI: 10.1016/j.jsams.2018.01.019.

Robinson LE., Stodden DF., Barnett LM., Lopes VP., Logan SW., Rodrigues LP., D’Hondt E. 2015. Motor Competence and its Effect on Positive Developmental Trajectories of Health. Sports Medicine (Auckland, N.Z.) 45:1273-1284. DOI: 10.1007/s40279-015-0351-6.

Saint-Maurice PF., Kim Y., Welk GJ., Gaesser GA. 2016. Kids are not little adults: what MET threshold captures sedentary behavior in children? European Journal of Applied Physiology 116:29-38. DOI: 10.1007/s00421-015-3238-1.

Sirard JR., Trost SG., Pfeiffer KA., Dowda M., Pate RR. 2005. Calibration and evaluation of an objective 
615

616

617

618

619

620

621

622

623

624

625

626

627

628

629

630

631

632

633

634

635

636

637

638

639

640

measure of physical activity in preschool children. Journal of Physical Activity \& Health 2:345-357. DOI: 10.1249/MSS.0b013e318206476e.

Stodden DF., Goodway JD., Langendorfer SJ., Roberton MA., Rudisill ME., Garcia C., Garcia LE. 2008. A Developmental Perspective on the Role of Motor Skill Competence in Physical Activity: An Emergent Relationship. Quest 60:290-306. DOI: 10.1080/00336297.2008.10483582.

Strong WB., Malina RM., Blimkie CJR., Daniels SR., Dishman RK., Gutin B., Hergenroeder AC., Must A., Nixon PA., Pivarnik JM., Rowland T., Trost S., Trudeau F. 2005. Evidence based physical activity for school-age youth. The Journal of Pediatrics 146:732-7. DOI: 10.1016/j.jpeds.2005.01.055.

Tanaka C., Reilly JJ., Huang WY. 2014. Longitudinal changes in objectively measured sedentary behaviour and their relationship with adiposity in children and adolescents: systematic review and evidence appraisal. Obesity Reviews 15:791-803. DOI: 10.1111/obr.12195.

Telama R., Yang X., Leskinen E., Kankaanpää A., Hirvensalo M., Tammelin T., Viikari JSA., Raitakari OT. 2014. Tracking of physical activity from early childhood through youth into adulthood. Medicine and Science in Sports and Exercise 46:955-62. DOI: 10.1249/MSS.0000000000000181.

Tikkanen O., Haakana P., Pesola AJ., Häkkinen K., Rantalainen T., Havu M., Pullinen T., Finni T. 2013. Muscle Activity and Inactivity Periods during Normal Daily Life. PLoS ONE 8:20-21. DOI: 10.1371/journal.pone.0052228.

Tikkanen O., Kärkkäinen S., Haakana P., Kallinen M., Pullinen T., Finni T. 2014. EMG, heart rate, and accelerometer as estimators of energy expenditure in locomotion. Medicine and Science in Sports and Exercise 46:1831-1839. DOI: 10.1249/MSS.0000000000000298.

Vigotsky AD., Halperin I., Lehman GJ., Trajano GS., Vieira TM. 2018. Interpreting signal amplitudes in surface electromyography studies in sport and rehabilitation sciences. Frontiers in Physiology 8. DOI: 10.3389/fphys.2017.00985.

Vähä-Ypyä H., Vasankari T., Husu P., Suni J., Sievänen H. 2015. A universal, accurate intensity-based classification of different physical activities using raw data of accelerometer. Clinical Physiology 
641 and Functional Imaging 35:64-70. DOI: 10.1111/cpf.12127.

642 WHO. 2010. Global recommendations on physical activity for health. World Health Organization:60.

643 DOI: $10.1080 / 11026480410034349$.

644

645 
646 FIGURE LEGENDS

647 Figure 1. Comparison of accelerometry (ACC) counts and electromyography (EMG) amplitude 648 during different PA tasks to children. Both ACC counts and EMG amplitude are normalized to 649 values obtained during individually chosen normal, preferred walking. All the ACC data in the 650 figure was analyzed using 1 second epoch. Data from both Study I and II were included in 651 walking $(\mathrm{n}=25)$, data from Study I: Balancing through Game of tag $(\mathrm{n}=11)$, Data from Study II, 652 Sitting through Jump for height $(\mathrm{n}=14$, samples averaged from 2 days). In Study II, EMG data 653 was missing from two participants.

654 Figure 2. Proportion of time in sedentary, light, moderate and vigorous activity intensity level in 655 various tasks compared with accelerometry (ACC) and electromyography (EMG). Mean data 656 from Study I, $\mathrm{n}=11$.

657 Figure 3. Bland-Altman plots showing differences in sedentary, light, moderate and vigorous 658 activity time between ACC and EMG. Positive values reflect ACC resulting in greater amount of 659 time and negative values indicate EMG provides greater amount of time spent at given PA level. 660 Significant linear correlations illustrate that heteroscedasticity was present at each category. 661 Figure 4. Comparing physical activity intensities during children's day when analyzed using 662 different cut-off points. EMG is shown as a comparison. $n=14$ (ACC), $n=10$ (EMG).

663 Figure 5. The effect of different epoch durations on physical activity intensities and comparison 664 to EMG assessed during children's daily life with and without exercise. The cut-off thresholds 665 by Evenson's method were used. $n=14(\mathrm{ACC}), \mathrm{n}=10(\mathrm{EMG})$. 


\section{TABLE LEGENDS}

667 Table 1. Average ( \pm SD, standard deviation) durations of each task in Study I and Study II. In 668 accelermetry analysis $1 \mathrm{~s}$ non-overlapping epochs were used in all tasks.

669 Table 2. Cut-off points for different physical activity intensities (15 s) in selected validation 670 studies. *The original cut-off points derived using $60 \mathrm{~s}$ epoch length were divided by 4 to be 671 comparable with $15 \mathrm{~s}$ values obtained in other studies.

672 Table 3. Sedentary time and physical activity levels during days with and without exercise using 673 accelerometry (ACC) and electromyography (EMG) methods. *ACC significantly different from 674 EMG method at given physical activity intensity, $\mathrm{p}<0.05$.

675

676

677

678

679

680

681

682

683 
Figure 1 (on next page)

Comparison of accelerometry (ACC) counts and electromyography (EMG) amplitude during different PA tasks to children.

Both ACC counts and EMG amplitude are normalized to values obtained during individually chosen normal, preferred walking. All the ACC data in the figure was analyzed using 1 second epoch. Data from both Study I and II were included in walking $(n=25)$, data from Study I: Balancing through Game of tag $(n=11)$, Data from Study II, Sitting through Jump for height ( $n=14$, samples averaged from 2 days). In Study II, EMG data was missing from two participants. 


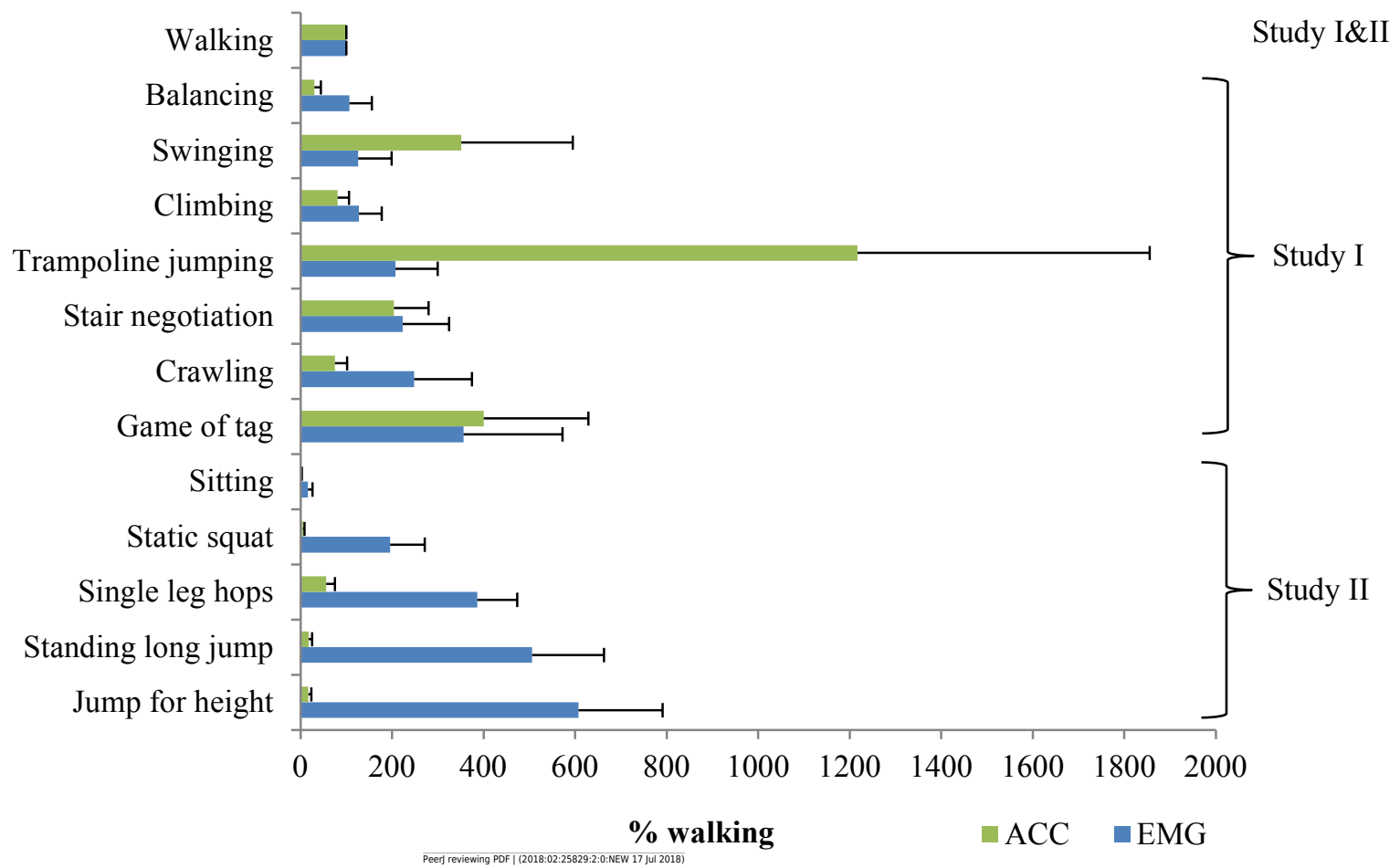




\section{Figure 2 (on next page)}

Proportion of time in sedentary, light, moderate and vigorous activity intensity level in various tasks compared with accelerometry (ACC) and electromyography (EMG).

Mean data from Study I, $\mathrm{n}=11$. 


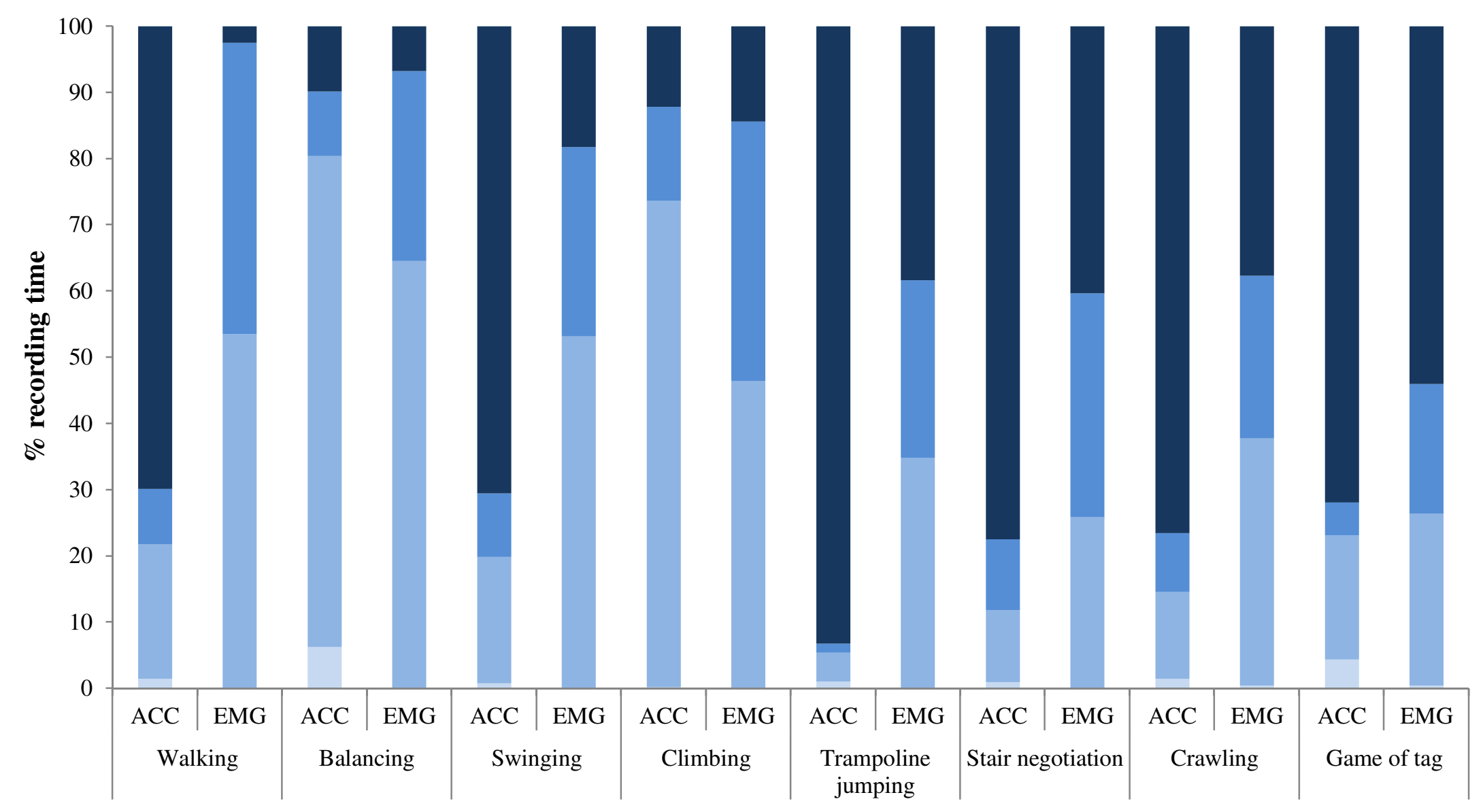

$\square$ Sedentary $\square$ Light activity $\square$ Moderate activity $\square$ Vigorous activity 


\section{Figure 3 (on next page)}

Bland-Altman plots showing differences in sedentary, light, moderate and vigorous activity time between ACC and EMG.

Positive values reflect ACC resulting in greater amount of time and negative values indicate EMG provides greater amount of time spent at given PA level. Significant linear correlations illustrate that heteroscedasticity was present at each category. 


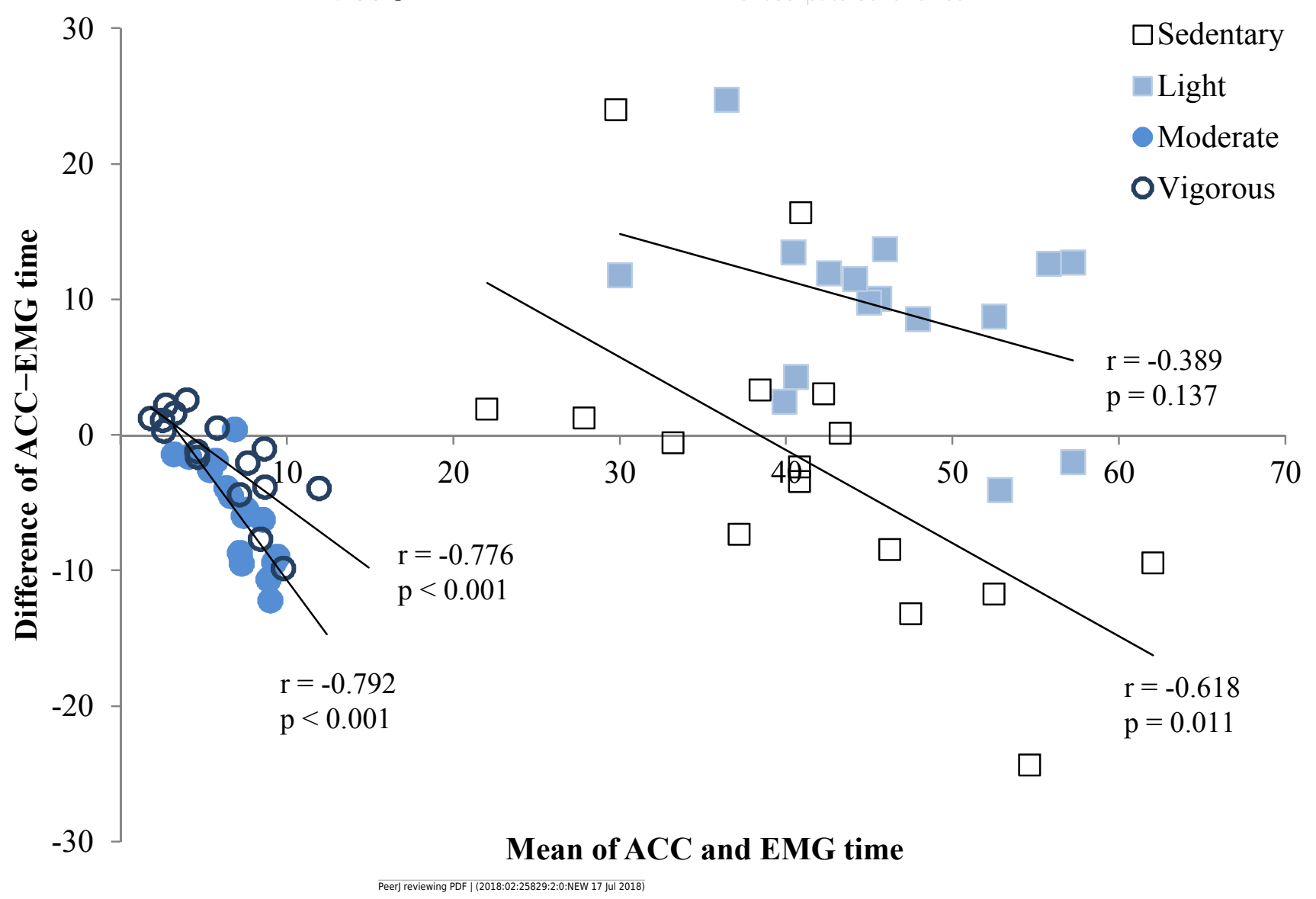




\section{Figure 4 (on next page)}

Comparing physical activity intensities during children's day when analyzed using different cut-off points.

EMG is shown as a comparison. $\mathrm{n}=14$ (ACC), $\mathrm{n}=10$ (EMG). 


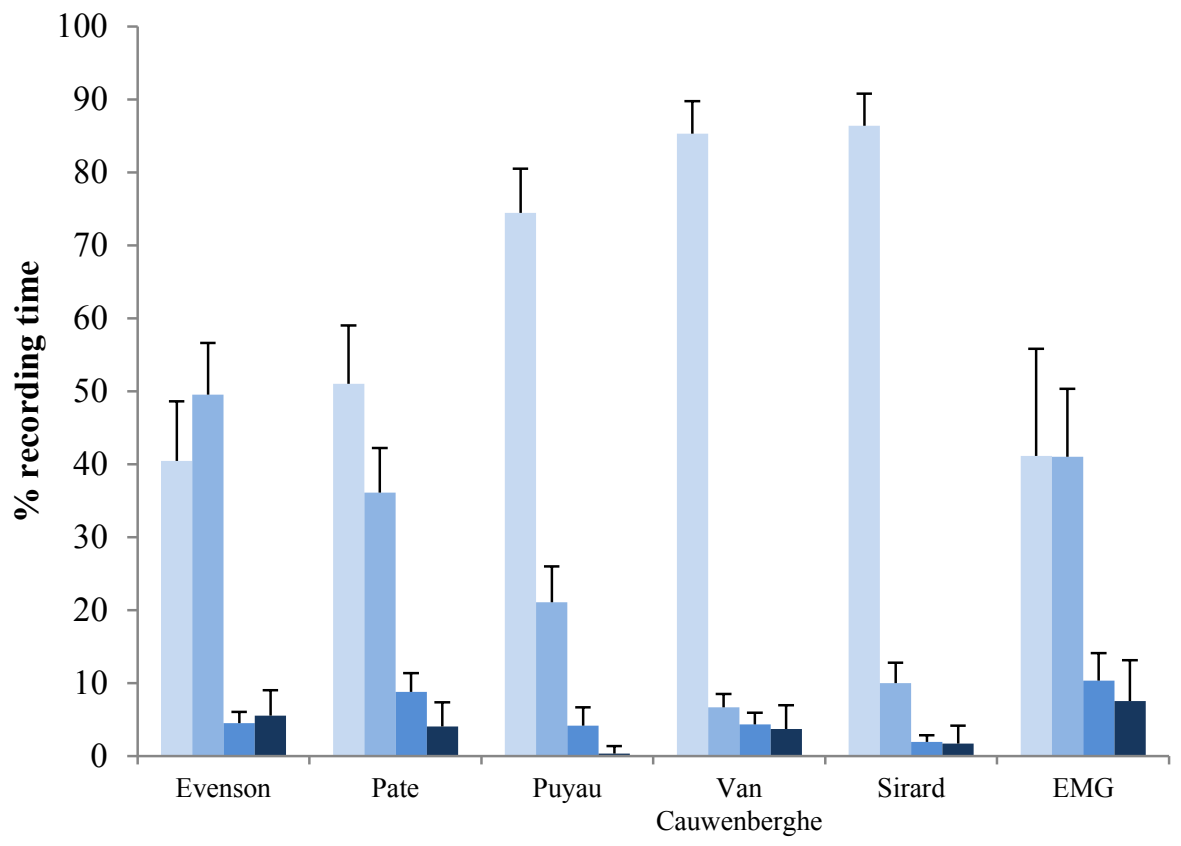

Sedentary

Light activity

Moderate activity

- Vigorous activity 


\section{Figure $\mathbf{5}$ (on next page)}

The effect of different epoch durations on physical activity intensities and comparison to EMG assessed during children's daily life with and without exercise.

The cut-off thresholds by Evenson's method were used. $n=14$ (ACC), $n=10$ (EMG). 


\section{Table $\mathbf{1}$ (on next page)}

Average ( $\pm S D$, standard deviation) durations of each task in Study I and Study II.

In accelermetry analysis $1 \mathrm{~s}$ non-overlapping epochs were used in all tasks. 


\begin{tabular}{llllll}
\hline \multicolumn{2}{c}{ Study I } & \multicolumn{4}{c}{ Study II } \\
Task & min:sec & SD & Task & min:sec & SD \\
\hline Walking & $00: 17$ & $00: 04$ & Walking & $00: 31$ & $00: 11$ \\
Stair negotiation & $00: 27$ & $00: 06$ & Sitting & $00: 31$ & $00: 15$ \\
Climbing & $00: 31$ & $00: 07$ & Static squat & $00: 21$ & $00: 11$ \\
Crawling & $00: 07$ & $00: 01$ & Single leg hops & $00: 04$ & $00: 01$ \\
Swinging & $01: 13$ & $00: 05$ & Jump for height & $00: 01$ & $00: 00$ \\
Balancing & $00: 15$ & $00: 13$ & Standing long jump & $00: 02$ & $00: 01$ \\
Trampoline jumping & $01: 52$ & $00: 04$ & & & \\
Game of tag & $00: 51$ & $00: 08$ & & & \\
\hline
\end{tabular}

1 


\section{Table 2 (on next page)}

Cut-off points for different physical activity intensities (15 s) in selected validation studies.

*The original cut-off points derived using $60 \mathrm{~s}$ epoch length were divided by 4 to be comparable with $15 \mathrm{~s}$ values obtained in other studies. 


\begin{tabular}{llll}
\hline Method & $\begin{array}{l}\text { Cut-off points: } \\
\text { Sedentary }\end{array}$ & $\begin{array}{l}\text { Light } \\
\text { intensity }\end{array}$ & $\begin{array}{l}\text { Moderate- to } \\
\text { Vigorous-intensity }\end{array}$ \\
\hline Evenson et al., 2008 & 12 & 508 & 719 \\
Pate et al., 2006; Pfeiffer et al., 2009 & 38 & 420 & 842 \\
Puyau et al., 2002* & 200 & 800 & 2050 \\
Van Cauwenberghe et al., 2011 & 373 & 585 & 881 \\
Sirard et al., 2005 & 399 & 891 & 1255 \\
\hline
\end{tabular}

1 


\section{Table 3(on next page)}

Sedentary time and physical activity levels during days with and without exercise using accelerometry (ACC) and electromyography (EMG) methods.

*ACC significantly different from EMG method at given physical activity intensity, $p<0.05$. 


\begin{tabular}{|c|c|c|}
\hline $\operatorname{ACC}(n=13)$ & Day with exercise & Day without exercise \\
\hline Duration of recording $(\min )$ & $526.1 \pm 34.5$ & $521.9 \pm 38.9$ \\
\hline Sedentary $(\%)$ & $38.7 \pm 7.1$ & $42.2 \pm 9.4$ \\
\hline Light activity (\%) & $50.7 \pm 6.7 *$ & $48.3 \pm 7.8^{*}$ \\
\hline Moderate activity (\%) & $4.7 \pm 1.0^{*}$ & $4.5 \pm 1.9 *$ \\
\hline Vigorous activity (\%) & $5.9 \pm 4.5$ & $5.0 \pm 2.4$ \\
\hline \multicolumn{3}{|l|}{ EMG $(n=7)$} \\
\hline Duration of recording (min) & $514.0 \pm 48.7$ & $513.5 \pm 47.1$ \\
\hline Muscle inactivity $(\%)$ & $45.5 \pm 12.9$ & $41.4 \pm 16.8$ \\
\hline Muscle light activity (\%) & $39.8 \pm 8.8$ & $40.9 \pm 10.9$ \\
\hline Muscle moderate activity (\%) & $9.3 \pm 3.1$ & $10.0 \pm 4.6$ \\
\hline Muscle vigorous activity (\%) & $5.5 \pm 3.4$ & $7.7 \pm 7.3$ \\
\hline
\end{tabular}

1 